\title{
Total mastectomy for phyllodes malignant tumor in an elderly patient
}

\author{
N. Bacalbașa ${ }^{1}$, Irina Bălescu², V. Brașoveanu ${ }^{3}$
}

Corresponding author:

Nicolae Bacalbasa, MD

Dimitrie Racovita Street, no. 2

Bucharest, Romania

E-mail: nicolae_bacalbasa@yahoo.ro

\author{
1"Carol Davila " University of Medicine and Pharmacy, Bucharest, Romania \\ 2"Ponderas Hospital" Bucharest, Romania \\ 3Department of General Surgery and Liver Transplantation "Dan Setlacec" \\ Fundeni Clinical Institute, Bucharest, Romania
}

\begin{abstract}
Phyllodes tumors are rare neoplasms accounting for less than $1 \%$ of all breast malignancies. Under this generic name more histopathological subgroups are included. Malignant phillodes tumors represent less than a quart of all phillodes tumors and are associated with a more aggressive biological behaviour with a higher tendency to both local and distant metastases. One of the most important prognostic factors in order to increase the overall ssurvival in these cases remains a wide excision of the primary tumor. We present a case of a 69 years old patient diagnosed with a large tumor deforming the left breast in whom the results of the punction-biopsy revealed a Phylodes malignant tumor. A total mastectomy was performed.
\end{abstract}

Key words: Phyllodes malignant tumor, elderly, total mastectomy

\section{INTRODUCTION}

Phyllodes tumors are rare neoplasms accounting for $<1 \%$ of all breast tumors [1], while malignant phyllodes tumors represent less than $25 \%$ of all phyllodes tumors. [1-3] Phyllodes tumors are formed by epithelial and stromal components bordered by mesenchymal cells; the malignancy of these tumors is given especially by the stromal elements.[4-7] Malignant transformation of a phyllodes tumor was first described in 1774 and fully classified in 1838 by Muller. $[8,9]$ Pathological aspects range from grade I to grade III malignant tumors, for the last cathegory the stromal component displaying a clear pattern of sarcoma. These kind of tumors are associated with a higher risk of recurrence and evolution towards an aggressive disease. [10,11] We present the case of a 69 year old patient who presented for the aparition of a large mass deforming the left breast in whom a total mastectomy was performed. The histopathological findings revealed a malignant Phyllodes tumor.

\section{CASE REPORT}

A 69 year old patient presented for the apparition of a large tumor deform- 
ing the left breast associated with nipple retraction. The tumor was first observed by the patient 3 months before and presented an accelerated growth associated with the presence of local pain. (figure 1) The fine needle aspiration revealed the presence of atypical spindle cells with up to 8 mitoses per 10 high power fields. The local clinical exam and the imagistic exams excluded the presence of enlarged axillar or intrathoracic lymph nodes. Surgery was performed and consisted in a total mastectomy due to the large volume of the tumor involving the central area of the breast. (figures 2, 3) The histopathological findings confirmed the results of preoperative fine needle punction; the resection margins of the specimen were free of tumoral invasion. The postoperative course was uneventful.

\section{DISCUSSIONS}

Although the highest proportion of breast malignancies is represented by adenocarcinomas originating from the mammary gland, tumor degeneration can occur in any kind of tissue present at the level of the breast giving birth to various kinds of tumors like angiosarcomas or leiomiosarcomas.[4] Phyllodes tumors (PT) are usually benign tumors which do not metastasize but might present a high rhythm of growth and a high capacity of recurrence. In rare cases they can develop a malignant transformation capable of producing both local recurrence and distant metastases. [5-7, 9] Initially recognised and diagnosed by their macroscopic fleshy aspect, in fact PT comprises a wide variety of tumours with mixed epithelial and benign or malignant mesenchymal proliferation. Malignant PT represents a specific sub-type of breast malignancies which is formed by both epithelial and connective tissue stroma. [4]

Due to the fact that sharp distinction between benign, borderline and malignant PT is sometimes difficult to be obtained, predictive factors for local recurrence or distant metastases are hard to be well established. [11] However, the gold standard in treating these tumors remains a complete surgical resection. [4] In different studies local recurrence is seen in $8-40 \%$ of cases while distant metastases are reported in $0-21 \%$ of cases, depending on the proportion of aggressive histopathological types of tumors comprised in each study. $[12,13]$

Histopathological studies classified PT in 3 groups, from grade I to grade III tumors -the last group being represented by malignant PT in which the stromal component displays a clear pattern of sarcoma. [4]

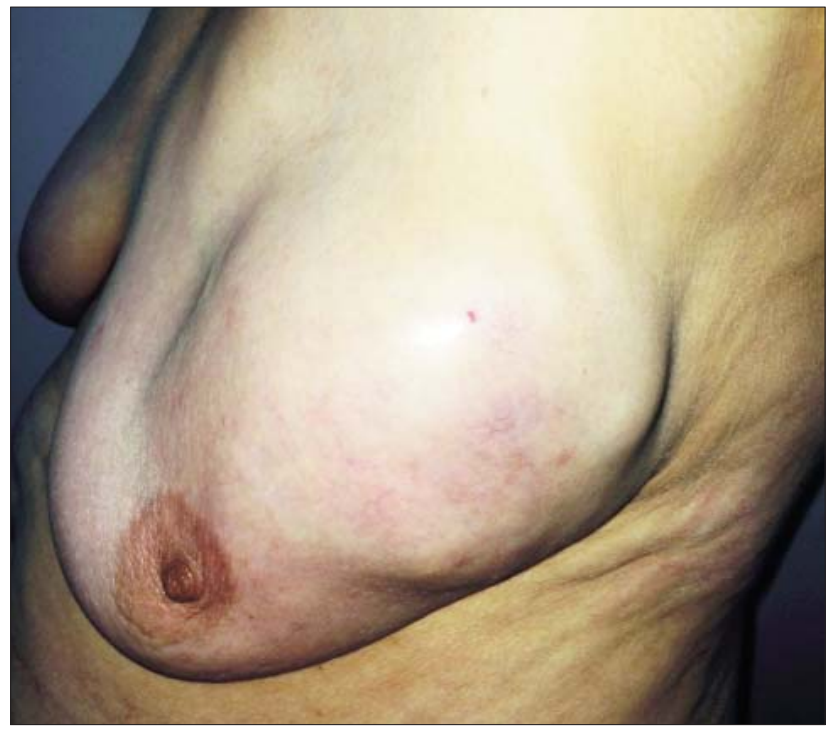

Figure 1 - large tumor entirely deforming the left breast

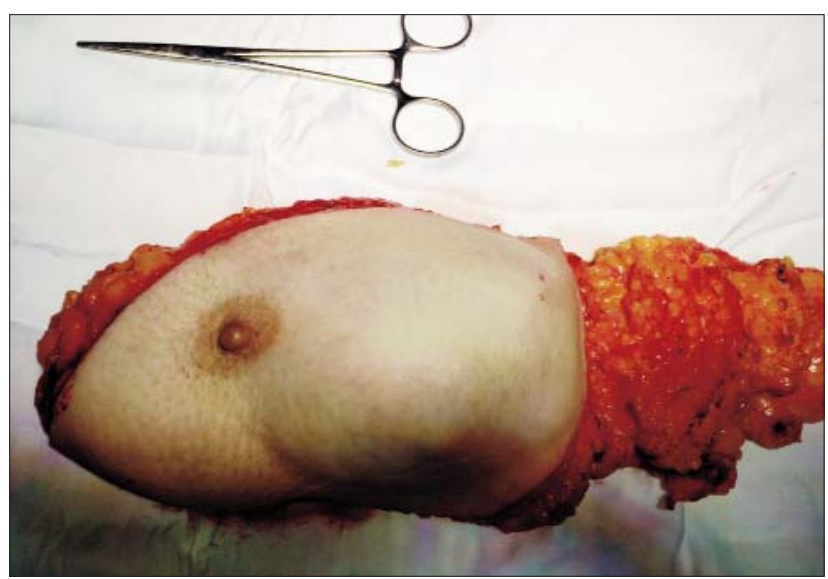

Figure 2 - the specimen of total mastectomy

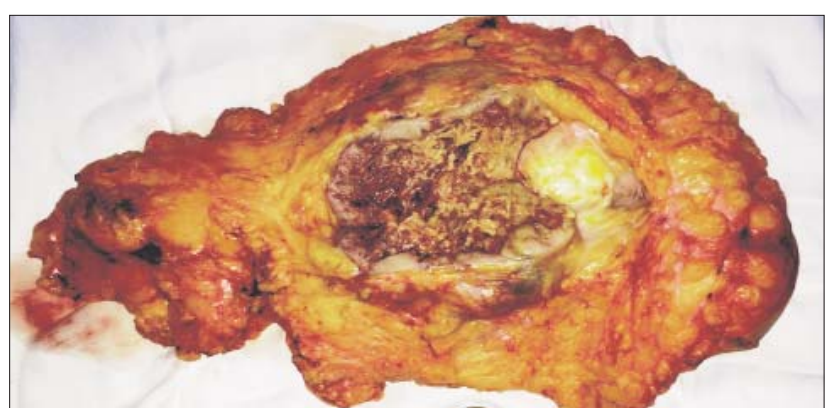

Figure 3 - the specimen presenting areas of fleshy tumor associated with necrosed and haemorrhagic areas

Confavreux et all conducted a study on 70 patients with primary breast sarcomas and PT. All patients excepting one were submitted to surgery first, the main performed procedures being mastectomy (46 cases) or wide excision ( 2 cases). The main histopathological 
types were primary breast sarcoma ( 44 cases), grade I PT (5 cases), grade II PT (5 cases) and grade III PT (16 cases). Axillary lymph node dissection was performed in 26 cases while only 2 cases presented positive lymph nodes at the microscopic evaluation. Postoperatively 17 patients underwent adjuvant chemotherapy- the main therapeutic protocol including anthracycline and alkylating agents while in 23 cases adjuvant radiotherapy was performed. At the end of adjuvant therapy complete response was achieved in 60 cases. When it comes about long term survival, it seems that the most important factors associated with long term survival were complete resection and histopathological type of tumor: patients who underwent RO resection reported a 3 year survival rate of $72 \%$ while this parameter significantly decreased in cases in whom R1-R2 resections were performed $(p=0,036)$. Lower histopathological grades of PT (I and II) reported a 3 year survival rate of $100 \%$ while in cases with PT grade III or with primary breast sarcomas the 3 year survival rate was only $57,6 \%(p=0,001)$. The overall survival rate at 5 years was $49 \%$. Half of the patients presented recurrent disease, 22 of them being diagnosed with recurrent tumors during the first 12 months. When it came about the site of the recurrence, 22 cases relapsed locally while 11 presented systemic disease and 2 experienced both types of relapse. The risk of recurrence could not be linked to the type of surgery or with the association of adjuvant oncologic treatment. This study revealed important similitudes between malignant PT and primary breast sarcomas and established significant prognostic factors but it was limited by the inclusion in the same study group of different hystopatological types. (4)

While in Confavreux's study there were no significant factors in order to predict tumoral recurrence, Eroglu et al demonstrated that tumour necrosis, stromal overgrowth, number of mitoses, stromal cellularity and atypia represent important factors in order to predict recurrence and Asoglu et al concluded that stromal overgrowth is the only relevant aspect in order to predict tumor recurrence. $[13,14]$

Although Confavreux et al failed to demonstrate any improvement of the overall survival in cases in which adjuvant radiation therapy was associated, some other authors sustain the benefit of this oncologic adjuvant treatment. $[15,16]$

The benefits of complete $\mathrm{R} O$ resection at the time of surgery has been widely demonstrated in clinical studies and remains one of the most important factors which increases the overall survival. [16-19]
In Popescu's study conducted between 1960 and 1989 in Fundeni Clinical Hospital, 19 cases with benign (15 cases) and malignant (4 cases) PT were included. Two of the cases initially diagnosed with benign tumors developped malignant recurrences. The authors concluded that in cases with malignant PT the treatment of choice is total mastectomy with wide resection of the tumor and surrounding tissues while in cases with initial benign disease a less extensive surgery (quadranectomy) associated with an adequate follow-up is the treatment of choice. [19]

In order to improve the long term results, some authors proposed association of neo-adjuvant chemoirradiation for patients diagnosed preoperatively with tumors larger than $5 \mathrm{~cm}$, but unfortunately only a increased disease free survival was obtained, while the overall survival remained the same. [20]

Ben Hassouna et al conducted a study on 106 patients diagnosed with PT, which were classified in 3 groups: benign ( 62 cases, $58.4 \%$ ), borderline (16 cases, $15 \%$ ), and malignant (28 cases, $26.4 \%$ ). Patients with malignant tumors underwent conservatory surgery in 9 cases and radical surgery in 19 cases. 5-year overall and disease-free survivals were $28.5 \%$ and $15.6 \%$ for patients with malignant PT who underwent conservative surgery versus $72.7 \%$ and $73.6 \%$ when the surgery was radical (mastectomy with or without axillary dissection) ( $p=0.12, p=0.022$ respectively). After $a$ mean follow up of 43 months, local recurrence was diagnosed in $17.8 \%$ cases $(5 / 28)$ with malignant PT, $31.2 \%$

(5/16) with borderline and 4.8\% (3/62) with benign PT. In four cases modifications of the initial hitopathological type appeared: 3 benign tumors transformed into bordeline tumors (in 2 cases), or into malignant tumor (1 case) while in a case initially diagnosed with borderline tumor a malignant PT was discovered at the moment of recurrence. Local recurrence was significantly increased in larger tumors, borderline and malignant forms presenting cytonuclear atypia, necrosis and hemorrhage, a higher number of mitosis or stromal overgrowth. As in the other mentioned studies, one of the most important factors for prolonged disease free survival was RO resections ( $p=0.01)$. When studying the apparition of distant metastases, they were seen in 8 cases: 7 patients were initially diagnosed with malignant PT while in the eigth case a borderline tumor had been found at the moment of primary surgery. The main sites involoved in developing distant metastases were bones ( $87,5 \%)$ and lungs (12,5\%) [21]. 


\section{CONCLUSIONS}

Under the general name of PT various histopathological types can be found, each category in part presenting different biological behaviour. Due to the rarity of these tumors there are no standard therapeutic protocols yet, but it is widely accepted that a complete $\mathrm{RO}$ resection is one of the most important factors associated both with prolonged disease free survival and overall survival. However further studied involving each histopathological subtype are still needed in order to establish which cases could benefit most from the association of adjuvant or neo-adjuvant oncologic treatment.

\section{REFERENCES}

1. Macdonald OK, Lee CM, Tward JD, Chappel CD, Gaffney DK. Malignant phyllodes tumor of the female breast: association of primary therapy with cause-specific survival from the Surveillance, Epidemiology, and End Results (SEER) program. Cancer 2006; 107(9):2127-2133.

2. Pietruszka M, Barnes L. Cystosarcoma phyllodes: a clinicopathologic analysis of 42 cases. Cancer 1978; 41(5):1974-1983.

3. Reinfuss M, Mitus J, Duda K, Stelmach A, Rys J, Smolak K. The treatment and prognosis of patients with phyllodes tumor of the breast: an analysis of 170 cases. In: 1996. pp. 910-916.

4. Confavreux C, Lurkin A, Mitton N, Blondet R, Saba C, Ranchere D, et al. Sarcomas and malignant phyllodes tumours of the breast--a retrospective study. Eur J Cancer 2006; 42(16):2715-2721.

5. Nabi J, Akhter SM, Authoy FN. A case of large phyllodes tumor causing "rupture" of the breast: a unique presentation. Case Rep Oncol Med 2013; 2013:871292.

6. Liang MI, Ramaswamy B, Patterson CC, McKelvey MT, Gordillo G, Nuovo GJ, et al. Giant breast tumors: surgical management of phyllodes tumors, potential for reconstructive surgery and a review of literature. World J Surg
Oncol 2008; 6:117.

7. Ang MK, Ooi AS, Thike AA, Tan P, Zhang Z, Dykema K, et al. Molecular classification of breast phyllodes tumors: validation of the histologic grading scheme and insights into malignant progression. Breast Cancer Res Treat 2011; 129(2):319-329.

8. Telli ML, Horst KC, Guardino AE, Dirbas FM, Carlson RW. Phyllodes tumors of the breast: natural history, diagnosis, and treatment. J Natl Compr Canc Netw 2007; 5(3):324-330.

9. White DS, Irvine TE. Rapidly progressive multifocal phyllodes tumour of the breast: A case report and review of the literature. Int J Surg Case Rep 2013; 4(10):901-903.

10. Tavassoli FA, Devilee P. Pathology and genetics of tumours of the breast and female genitale organs. IARC Press Lyon 2003.

11. Cheng-Chiao Huang, Tsang-Pai Liu, Shih-Ping Cheng, Yuan-Ching Chang. Surgical Treatment of Phyllodes Tumor of the Breast with the Trend. J Exp Clin Med 2014; 6(5).

12. Parker SJ, Harries SA. Phyllodes tumours. Postgrad Med J 2001; 77(909): 428-435.

13. Eroglu E, Irkkan C, Ozsoy M, Eroglu F. Phyllodes tumor of the breast: case series of 40 patients. Eur J Gynaecol Oncol 2004; 25(1):123-125.

14. Asoglu 0, Ugurlu MM, Blanchard K, Grant CS, Reynolds C, Cha SS, et al. Risk factors for recurrence and death after primary surgical treatment of malignant phyllodes tumors. Ann Surg Oncol 2004; 11(11):1011-1017.

15. Joshi SC, Sharma DN, Bahadur AK, Maurya R, Kumar S, Khurana N. Cystosarcoma phyllodes: our institutional experience. Australas Radiol 2003; 47(4):434-437.

16. Pandey M, Mathew A, Kattoor J, Abraham EK, Mathew BS, Rajan B, et al. Malignant phyllodes tumor. Breast J 2001; 7(6):411-416.

17. Trent II JC, Benjamin RS, Valero V. Primary soft tissue sarcoma of the breast. Curr Treat Options Oncol 2001; 2(2):169-176.

18. Khan SA, Badve S. Phyllodes tumors of the breast. Curr Treat Options Oncol 2001; 2(2):139-147.

19. Popescu I, Serbanescu M, Ivaschescu C. Phylloides tumours of the breast. A clinicopathologic study of 19 cases. Zentralbl Chir 1991; 116(5):327336.

20. Gutman H, Pollock RE, Ross Ml, Benjamin RS, Johnston DA, Janjan NA, et al. Sarcoma of the breast: implications for extent of therapy. The M. D. Anderson experience. Surgery 1994; 116(3):505-509.

21. Ben HJ, Damak T, Gamoudi A, Chargui R, Khomsi F, Mahjoub S, et al. Phyllodes tumors of the breast: a case series of 106 patients. Am J Surg 2006; 192(2):141-147. 